\title{
Plasma copeptin as biomarker of disease progression and prognosis in cirrhosis
}

\author{
Elsa Solà ${ }^{1,2,3, *, \dagger}$, Annarein J.C. Kerbert ${ }^{5, \dagger}$, Hein W. Verspaget ${ }^{5}$, Rebeca Moreira ${ }^{1,2,3}$, Elisa Pose ${ }^{1,2,3}$, \\ Pablo Ruiz ${ }^{1,2,3}$, Raquel Cela ${ }^{1,2,3}$, Manuel Morales-Ruiz ${ }^{2,3,6}$, Eva López ${ }^{1,2,3}$, Isabel Graupera ${ }^{1,2,3}$, \\ Cristina Solé ${ }^{1,2,3}$, Patricia Huelin ${ }^{1,2,3}$, Alex Amorós Navarro ${ }^{7}$, Xavier Ariza ${ }^{1,2,3}$, Rajiv Jalan ${ }^{8}$, \\ Núria Fabrellas ${ }^{2,4}$, Daniel Benten ${ }^{9}$, Gloria de Prada ${ }^{1,2,3}$, François Durand ${ }^{10}$, Wladimiro Jimenez ${ }^{2,3,6}$, \\ Johan J. van der Reijden ${ }^{5}$, Javier Fernandez ${ }^{1,2,3}$, Bart van Hoek ${ }^{5}$, Minneke J. Coenraad ${ }^{5,}$, \\ Pere Ginès ${ }^{1,2,3, *}$
}

\begin{abstract}
${ }^{1}$ Liver Unit, Hospital Clínic, University of Barcelona, Barcelona, Spain; ${ }^{2}$ Institut d'Investigacions Biomèdiques August Pi i Sunyer (IDIBAPS), Barcelona, Spain; ${ }^{3}$ Centro de Investigación Biomédica en Red de Enfermedades Hepáticas y Digestivas (CIBEREHD), Spain; ${ }^{4}$ School of Nursing, University of Barcelona, Spain; ${ }^{5}$ Department of Gastroenterology-Hepatology, Leiden University Medical Center, Leiden, The Netherlands; ${ }^{6}$ Biochemistry and Molecular Genetics Department, Hospital Clínic, University of Barcelona, Barcelona, Spain; ${ }^{7}$ EASL-CLIF Data Center, Barcelona, Spain; ${ }^{8}$ Liver Failure Group, UCL Institute of Liver and Digestive Health, UCL Medical School, Royal Free Hospital, London, United Kingdom; ${ }^{9}$ Department of Medicine, University Medical Center Hamburg-Eppendorf, Hamburg, Germany;

${ }^{10}$ Hepatology and Liver Intensive Care Unit, Hospital Beaujon, Clichy, France
\end{abstract}

Background \& Aims: Research on vasopressin (AVP) in cirrhosis and its role in the assessment of prognosis has been hindered by the difficulty of measuring AVP levels accurately. Copeptin, a 39aminoacid glycopeptide, is released from the neurohypophysis together with AVP. Copeptin could have a role as biomarker of prognosis in cirrhosis as it may reflect circulatory dysfunction. The aim of this study is to investigate the role of copeptin as biomarker of disease progression and prognosis in cirrhosis.

Methods: This prospective study is divided in 2 study protocols including 321 consecutive patients. Plasma copeptin levels were measured in all patients at study inclusion. Protocol 1: to investigate the relationship of copeptin with kidney and circulatory function (56 patients). Protocol 2: to investigate the relationship between copeptin and prognosis, as assessed by the development of complications of cirrhosis or mortality at 3 months (265 patients admitted to hospital for complications of cirrhosis).

Results: Patients with decompensated cirrhosis showed significantly higher plasma copeptin levels compared to those of patients with compensated cirrhosis. Copeptin levels had a significant positive correlation with model for end-satge liver disease (MELD) score, AVP, endogenous vasoconstrictor systems, and kidney function parameters. Patients developing complications of cirrhosis or mortality had significantly higher plasma copeptin levels compared to those of the remaining patients. Plasma

Keywords: Copeptin; Biomarker; Cirrhosis; Circulatory dysfunction; Prognosis. Received 9 July 2015; received in revised form 28 June 2016; accepted 4 July 2016; available online 12 July 2016

t5 Guest Editor: Didier Samuel.

* Corresponding author. Address: Liver Unit, Hospital Clínic, University of Barcelona, Villarroel 170, 08036 Barcelona, Spain. Tel.: +34932271713.

E-mail address: esola@clinic.cat (E. Solà).

$\dagger$ These authors contributed equally as joint first authors.

\$ These authors share senior authorship. copeptin levels were an independent predictive factor of both the development of complications and mortality at 3 months. This was confirmed in a validation series of 120 patients.

Conclusions: Copeptin is a novel biomarker of disease progression and prognosis in cirrhosis.

Lay summary: Copeptin is a fragment of the vasopressin precursor, a hormone that is known to be increased in patients with cirrhosis and that plays a role in the development of complications of the disease. Vasopressin is difficult to measure, but copeptin is a more stable molecule and is easier to measure in blood. Solà and Kerbert and colleagues have shown in a series of 361 patients that copeptin is markedly increased in patients with cirrhosis who develop complications during the following 3 months, compared to those patients who do not develop complications. Moreover, copeptin correlates with prognosis.

(c) 2016 European Association for the Study of the Liver. Published by Elsevier B.V. All rights reserved.

\section{Introduction}

Natural history of cirrhosis is characterized by decompensation of the disease, which is associated with high morbidity and mortality [1]. Clinical decompensation is mainly related to a progressive increase in portal hypertension and circulatory dysfunction. In the early stages of the disease when cirrhosis is compensated and patients are still asymptomatic, there is only a slight decrease in systemic vascular resistance and arterial effective blood volume and blood pressure are maintained within normal levels $[2,3]$. As disease progresses, patients develop clinical decompensation associated with worsening circulatory dysfunction. In this stage of the disease, circulatory dysfunction is 


\section{JOURNAL OF HEPATOLOGY}

characterized by marked decrease in systemic vascular resistance due to an intense splanchnic vasodilation that leads to a reduction in effective arterial blood volume. In order to maintain arterial pressure within normal limits there is a marked activation of endogenous vasoconstrictor systems, such as renin-angiotensinaldosterone system, and sympathetic nervous system. In more advanced stages of the disease, there is non-osmotic secretion of vasopressin (AVP). The activation of these vasoconstrictor systems helps improve circulatory function but has negative effects on kidney function leading to renal sodium and solute-free water retention, which is associated with development of ascites and dilutional hyponatremia. Eventually, there is an intense kidney vasoconstriction leading to hepatorenal syndrome [2,3]. Therefore, considering the pathophysiology of disease progression, AVP could theoretically represent a biomarker of advanced disease, clinical decompensation and prognosis in cirrhosis. However, research on AVP in cirrhosis and its role in the pathophysiology of the disease has been hindered by the difficulty of measuring AVP levels accurately. The accurate measurement of plasma AVP levels is difficult because of its short half-life and methodological issues [4-6].

Copeptin, a 39-aminoacid glycopeptide, is released from the neurohypophysis stoichiometrically with AVP, within the precursor preprovasopressin [7-9]. The precursor consists of a signal peptide, AVP, neurophysin-II, and copeptin. In contrast to AVP, copeptin is a non-functional peptide which is very stable and easier to measure [7-9]. There is evidence suggesting that copeptin is a good surrogate marker of AVP, as there is a good correlation between AVP and copeptin levels [10-13]. The development of a reliable immunoassay to measure copeptin allows investigation into the role of AVP as a biomarker in clinical practice [10]. Moreover, assessment of plasma copeptin levels may help understand the mechanism(s) of the increased release of AVP from the neurohypophysis into the circulation and its pathogenic role in cirrhosis.

Copeptin has been shown to be associated with prognosis in several diseases such as heart failure, chronic respiratory diseases and critically-ill patients [14-20]. However, information in cirrhosis is scarce [21]. Therefore, the aim of our study was to assess the role of copeptin as biomarker of circulatory derangement, disease progression and acute decompensation in patients with cirrhosis. Moreover, the study was aimed at evaluating the usefulness of copeptin as a biomarker to predict prognosis in patients with cirrhosis including the validation of the prediction model in an independent cohort of patients.

\section{Patients and methods}

Study design

This was a prospective study that included 321 consecutive patients with cirrhosis seen at the Liver Unit of Hospital Clinic of Barcelona from February 2011 to February 2013. The study was divided in two different protocols that were conducted simultaneously. Patients seen at the outpatient clinic were considered for protocol 1 and patients admitted to hospital for complications of cirrhosis were included in protocol 2 . The only patients excluded from the study were: (1) patients with chronic kidney disease treated with hemodialysis before admission, (2) patients with previous liver or kidney transplantation, (3) patients with hepatocellular carcinoma beyond Milan criteria, (4) patients with advanced chronic respiratory or heart disease and (5) patients with severe extrahepatic diseases with poor short-term prognosis. All patients signed a written informed consent document and gave permission for samples to be used in the study following current national and institutional guidelines for sample storage and usage for research purposes. The study was approved by the Institutional Review Board of our center.

Protocol 1

Fifty-six patients seen at the liver clinic were included, 18 (32\%) with compensated cirrhosis and 38 (68\%) with decompensated cirrhosis. The specific aim of this first part of the study was to investigate the relationship between plasma copeptin levels and kidney and circulatory function and the stages of the disease.

Demographical, clinical and analytical data were collected from all patients at study inclusion. Analytical data for standard liver and kidney function tests and hematology were obtained from blood tests taken as part of usual clinical assistance. In addition, $20 \mathrm{ml}$ of blood were obtained to measure plasma levels of copeptin and endogenous vasoconstrictor systems: plasma renin activity (PRA) and the plasma concentrations of vasopressin, aldosterone, and noradrenaline.

Protocol 2

Two-hundred and sixty-five consecutive patients admitted to hospital for complications of cirrhosis were prospectively included in the study. The specific aim of this part of the study was to assess the relationship between plasma copeptin levels and complications of cirrhosis and survival.

Demographical, clinical and analytical data were collected from all patients at study inclusion. Analytical data for standard liver and kidney function tests and hematology were obtained from blood tests taken for usual clinical assistance. In addition, $10 \mathrm{ml}$ of blood were obtained to measure plasma levels of copeptin. Patients were followed up for a 3-month period and complications of cirrhosis occurring both during hospitalization and after discharge from hospital were recorded. Complications were categorized as ascites, hepatic encephalopathy (HE), bacterial infections, gastrointestinal (GI) bleeding, hyponatremia, and kidney failure.

\section{Definitions}

Ascites was defined as the development of de novo ascites requiring initiation of diuretic treatment or worsening of previous existent ascites requiring an increase in diuretic treatment dose or large-volume paracentesis. Hepatic encephalopathy was defined as the development of grade $\geqslant 2 \mathrm{HE}$ that required hospital admission. GI bleeding was defined as portal hypertension related bleeding requiring hospital admission. Hyponatremia was defined as a decrease in serum sodium levels of $5 \mathrm{mEq} / \mathrm{L}$ with respect to baseline with a final value $<130 \mathrm{mEq} / \mathrm{L}$. Kidney failure was defined as an increase of serum creatinine $>50 \%$ with respect to baseline with a final value $>1.5 \mathrm{mg} / \mathrm{dl}[22,23]$. Finally, bacterial infections were defined as reported in detail elsewhere [24]

Analytical methods

Blood samples for plasma copeptin levels were processed within $2 \mathrm{~h}$ after blood extraction. Samples were centrifuged at $3000 \mathrm{~g}$ for $15 \mathrm{~min}$ at $4{ }^{\circ} \mathrm{C}$ and the supernatant stored at $-80{ }^{\circ} \mathrm{C}$ until analysis. Copeptin was measured using a sandwich immunoluminometric assay using two polyclonal antibodies to the aminoacid sequences 132-147 and 149-164 in the C-terminal region of the vasopressin precursor (BRAHMS Copeptin US KRYPTOR, Thermofisher Scientific, Hennigsdorf, Germany). The assay is very sensitive, with an analytical detection limit of $1.7 \mathrm{pmol} / \mathrm{L}$. Moreover, it has a good precision with an interlaboratory coefficient of variation $(\mathrm{CV})<20 \%$ for all copeptin concentrations [10].

Measurement of plasma levels of vasopressin, PRA, aldosterone and noradrenaline was performed using previously described methods [25,26]. Briefly, $10 \mathrm{ml}$ of blood were obtained from patients after a $6 \mathrm{~h}$ fasting period and after maintenance of supine position for $1 \mathrm{~h}$ before blood sampling. All vasoactive hormones were analyzed using radioimmunoassay (Bühlmann, IBL International and Diiasorin, respectively) with intra-assay coefficient of variation $<7 \%$ and interassay coefficient of variation $<15 \%$ for all the assays.

\section{Statistical analysis}

Results for continuous variables are expressed as mean \pm standard deviation or median (interquartile range). Categorical variables are expressed as number and percentage. Comparisons between groups were made with standard statistical tests. Complications and survival probability curves were calculated with the Kaplan-Meier method and compared with log-rank test. When analyzing threemonth mortality, both mortality and liver transplantation were considered as an event. To assess the variables independently associated with complications 


\section{Research Article}

of cirrhosis or 3-month mortality, a logistic regression model was performed. The model was fitted applying a forward stepwise selection method with $p$ in $=0.05$ and $p$ out $=0.1$. In order to avoid collinearity with Child-Pugh and MELD scores, individual variables included in these scores were not included in the model An internal validation of the prediction model of mortality was performed with the use of the bootstrapping technique. Moreover, the performance of the mode was evaluated in an external validation with an independent series including patients from other centers. Variables with skewed distribution, such as copeptin were log-transformed before being included in the analysis. Since the number of missing data was very low, particularly for the main variables, no imputation of missing data was used and a complete cases analysis was performed. All statistical analyses were performed using SPSS 20.0 software. The significance level for all statistical tests was set at 0.05 two-tailed. The results of the study are reported following the TRIPOD (transparent reporting of a multivariable prediction model for individual prognosis or diagnosis) Statement recommendations [27].

\section{Results}

\section{Study protocol 1}

\section{Characteristics of the patient population}

Out of the 56 patients included in this first part of the study, 64\% were male and the main etiology of cirrhosis was alcohol (54\%). Patients had a moderate impairment in liver function as reflected by slightly increased serum bilirubin levels $(3.3 \pm 5 \mathrm{mg} / \mathrm{dl})$ and a mean MELD score of 11 . Baseline demographical, clinical and analytical data of this series of patients are shown in Supplementary Table 1.

Plasma copeptin levels and relationship with liver, kidney and circulatory function

In the overall series, median plasma copeptin levels were $12 \mathrm{pmol} / \mathrm{L}$ (IQR 5-26). Plasma copeptin levels were not related to age, gender, etiology of cirrhosis or the presence of active alcoholism at the time of inclusion. Plasma copeptin levels were markedly higher in patients with decompensated cirrhosis compared to those in patients with compensated cirrhosis (median 21 [IQR 8.1-49.5] vs. 4.5 [2.9-8.4] pmol/L, respectively; $p<0.0001$ ) (Fig. 1A). Moreover, when patients were stratified
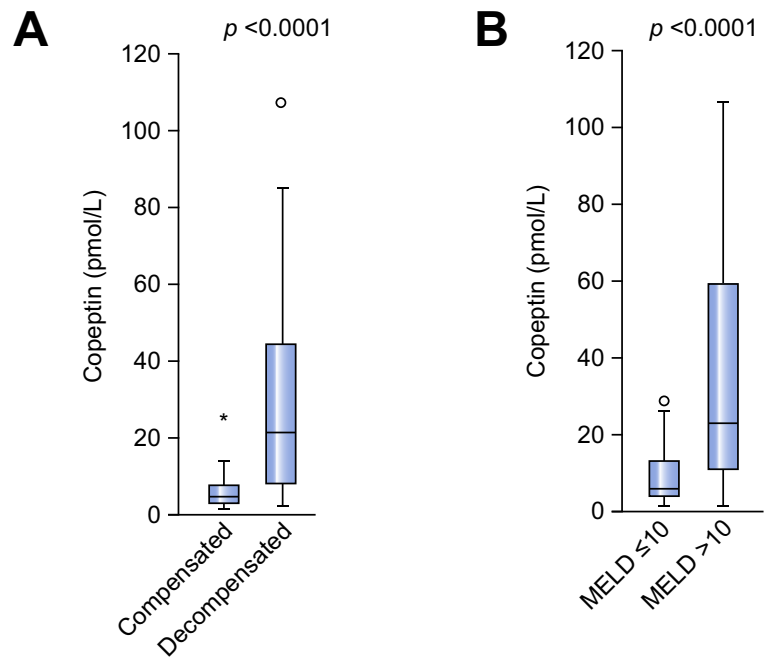

Fig. 1. Plasma copeptin levels according to disease status in patients included in protocol 1. (A) Plasma copeptin levels in patients categorized according to compensated or decompensated cirrhosis. (B) Plasma copeptin levels in patients categorized according to median MELD score of the whole series. according to Child-Pugh class, plasma copeptin levels were significantly higher in patients with Child-Pugh class B (10.7 [6.424.1]) and C (59.9 [19.3-75.7] pmol/L) compared to patients with Child-Pugh A (4.6 pmol/L [3.1-12.4]) $(p=0.001)$. Similarly, when patients were categorized according to MELD score, plasma copeptin levels were significantly higher in patients with MELD score $>10$ (median value of the series), compared to patients with MELD score $\leqslant 10$ (23 [9.7-60.2] vs. 6.5 [4.1-13.8], respectively; $p<0.0001$ ) (Fig. 1B). Indeed, there was a positive and significant correlation between copeptin and MELD score $(r=0.677$; $p<0.0001$ ).

We then analyzed the relationship between plasma copeptin levels and kidney and circulatory function. There was a positive and statistically significant correlation between plasma copeptin levels and kidney function, as estimated by serum creatinine concentration $(r=0.603 ; p<0.0001)$. Besides, copeptin was negatively correlated with mean arterial pressure $(r=-0.399$; $p=0.003$ ). Moreover, there was a significant positive correlation between plasma copeptin and endogenous vasoconstrictor systems as reflected by correlation with PRA $(r=0.445 ; p=0.001)$, aldosterone $(\mathrm{r}=0.487 ; p<0.0001)$ and noradrenaline $(\mathrm{r}=0.459$; $p=0.001$ ).

Finally, as copeptin is the C-terminal fragment of AVP we also analyzed the relationship between plasma copeptin and vasopressin, serum sodium levels and plasma osmolality. In keeping with results from previous studies in other disease conditions, there was a significant and positive correlation between plasma copeptin and vasopressin levels $(r=0.654 ; p<0.0001)$. Moreover there was a statistically significant inverse correlation with serum sodium levels $(r=-0.462, p<0.0001)$; however, there was no significant correlation with plasma osmolality $(\mathrm{r}=-0.109 ; p=0.438)$. When patients were categorized according to baseline serum sodium levels, patients with hyponatremia had significantly higher plasma copeptin levels compared to those with normal serum sodium concentration (24.2 [7.764.3] vs. 10.9 [4.5-23] pmol/L, respectively), $p=0.026$.

\section{Study protocol 2}

Characteristics of the patient population

Demographical and clinical data, and liver and kidney function tests of the 265 patients included in this part of the study are shown in Table 1. Patients had moderately-severe impairment of liver function as reflected by high serum bilirubin, mean MELD score of 17 and Child-Pugh score of 9. Patients had a moderate impairment of kidney function with mean serum creatinine of $1.3 \mathrm{mg} / \mathrm{dl}$.

Plasma copeptin levels and clinical outcomes

In this series of patients, median plasma copeptin levels were $14 \mathrm{pmol} / \mathrm{L}$ (IQR 7.3-33). Out of the 265 patients enrolled, 206 patients (78\%) developed complications of cirrhosis either during hospitalization or after discharge from hospital. The most frequent complication was bacterial infections in 121 patients (46\%), followed by HE (38\%), kidney failure (33\%), ascites (25\%), hyponatremia (17\%) and GI bleeding (11\%). Patients developing complications of cirrhosis during follow-up had significantly higher plasma copeptin levels compared to those patients patients who did not develop complications during follow-up (17 [9-38] vs. 8.6 [6-17] $\mathrm{pmol} / \mathrm{L}$, respectively; $p<0.0001$ ). Patients developing any of the individual complications of 
Table 1. Demographic and clinical data and liver and kidney function tests of the 265 patients included in protocol 2.

\begin{tabular}{|c|c|c|}
\hline Variable & & \\
\hline Age (years) & $60 \pm 11$ & $(21-85)$ \\
\hline Gender, male & $176(66 \%)$ & \\
\hline \multicolumn{3}{|l|}{ Etiology of cirrhosis: } \\
\hline Alcohol & $114(43 \%)$ & \\
\hline Hepatitis C & $91(34 \%)$ & \\
\hline Other & $60(23 \%)$ & \\
\hline Active alcoholism & $77(29 \%)$ & \\
\hline Previous ascites & $189(71 \%)$ & \\
\hline Previous hepatic encephalopathy & $96(36 \%)$ & \\
\hline \multicolumn{3}{|l|}{ Cause of admission } \\
\hline Bacterial infections & $82(26 \%)$ & \\
\hline Ascites & $71(22 \%)$ & \\
\hline Hepatic encephalopathy & $31(10 \%)$ & \\
\hline GI bleeding & $24(8 \%)$ & \\
\hline Kidney failure & $15(5 \%)$ & \\
\hline Other & $42(13 \%)$ & \\
\hline Shock at admission & $24(8 \%)$ & \\
\hline Serum bilirubin (mg/dl) & $5.9 \pm 7.7$ & $(0.3-40)$ \\
\hline Serum albumin (g/L) & $27 \pm 5$ & $(17-44)$ \\
\hline INR & $1.7 \pm 0.5$ & $(0.9-4.4)$ \\
\hline Child-Pugh score & $9 \pm 2$ & $(5-14)$ \\
\hline MELD score & $17 \pm 8$ & $(7-40)$ \\
\hline Serum creatinine (mg/dl) & $1.3 \pm 0.9$ & $(0.4-9.2)$ \\
\hline Serum sodium (mEq/L) & $135 \pm 5$ & $(114-148)$ \\
\hline Leukocyte count $\left(10^{9}\right.$ cells/L) & $6.9 \pm 5$ & $(0.6-39)$ \\
\hline Mean arterial pressure $(\mathrm{mmHg})$ & $84 \pm 14$ & $(40-131)$ \\
\hline Copeptin $(p m o l / L)^{\delta}$ & $14(7-32)$ & \\
\hline Vasopressin $(\mathrm{ng} / \mathrm{L})^{\delta}$ & $2.1(1.4-2.9)$ & \\
\hline
\end{tabular}

Data are expressed as mean \pm SD and ranges or number and percentages, except for copeptin and vasopressin which are expressed as median (interquartile range).

${ }^{8}$ Median plasma copeptin levels in a series of 20 healthy volunteers were 8.5 pmol/L (IQR 3.9-14.5). Normal values for vasopressin levels are 1.5-3.3 ng/L.

cirrhosis, except for ascites and hyponatremia, had significantly higher plasma copeptin levels compared to those of patients who did not develop these complications (Table 2).

In univariate analysis, besides plasma copeptin levels, other variables significantly associated with development of complications of cirrhosis were: prior history of ascites, HE or infections, serum bilirubin, international normalized ratio (INR), serum creatinine, serum sodium, AVP levels, and MELD and Child-Pugh scores (Supplementary Table 2). Patients with plasma copeptin levels $>14 \mathrm{pmol} / \mathrm{L}$ (median value of the whole series) had a probability of developing complications during follow-up significantly higher than that of patients with plasma copeptin levels $\leqslant 14 \mathrm{pmol} / \mathrm{L}$ (78\% vs. 60\%, respectively; $p=0.005$ ). A logistic regression model showed that plasma copeptin levels together with serum sodium were independent predictive factors of development of complications of cirrhosis during follow-up (AUROC 0.723; 95\% CI [0.650-0.796]) (Table 3).

Out of the 265 patients, 63 patients died (24\%) and 16 were transplanted (6\%) during the 3-month follow-up period. Patients who died during follow-up showed significantly higher plasma copeptin levels compared to those of patients who were alive at the end of follow-up (37.7 [12-63] vs. 11.8 [6-23], respectively; $p<0.0001$ ). Fig. 2 shows the probability of survival in all

\section{JOURNAL OF HEPATOLOGY}

Table 2. Plasma copeptin levels according to development of complications of cirrhosis during follow-up.

\begin{tabular}{llll}
\hline Complications of cirrhosis & Yes & No & $p$ value \\
\hline Any complication $(n=206)$ & $17(9-38)$ & $8.6(6-17)$ & 0.007 \\
Bacterial infections $(n=121)$ & $17(9-42)$ & $11(6-21)$ & $<0.0001$ \\
Hepatic encephalopathy $(n=101)$ & $19(10-40)$ & $11(6-21)$ & 0.001 \\
Kidney failure $(n=86)$ & $31(16-52)$ & $10(6-19)$ & $<0.0001$ \\
Ascites $(n=66)$ & $15(8-30)$ & $12(7-29)$ & 0.637 \\
Hyponatremia $(n=46)$ & $13(7-38)$ & $13(7-26)$ & 0.567 \\
Gl bleeding $(n=28)$ & $28(10-75)$ & $12(7-27)$ & $<0.0001$ \\
\hline
\end{tabular}

Plasma copeptin levels are in pmol/L and are represented as median (interquartile range).

Number in brackets after each complication represent the number of patients who developed each complication during the 3-month follow-up period.

Table 3. Independent predictive factors of development of complications of cirrhosis during follow up. Multivariate analysis.

\begin{tabular}{llll}
\hline Variable & OR & $95 \% \mathrm{Cl}$ & $p$ value \\
\hline Copeptin & 1.575 & $1.102-2.252$ & 0.013 \\
Serum sodium & 0.894 & $0.825-0.970$ & 0.007 \\
\hline AUROC $0.723 .95 \%$ & Cl $(0.650-0.796)$ & &
\end{tabular}

AUROC 0.723; 95\% CI (0.650-0.796).

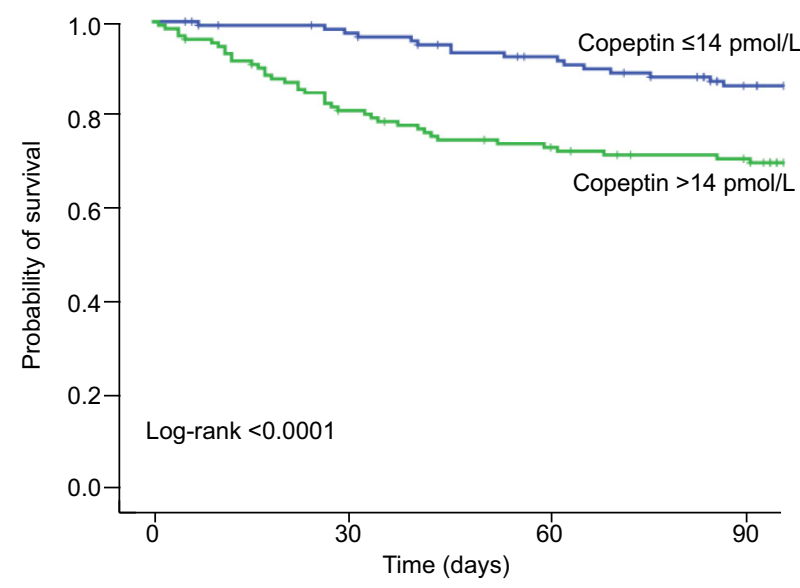

Fig. 2. Kaplan-Meier curves showing probability of survival during the 3-month follow-up period in patients included in protocol 2. Categorized according to median baseline plasma copeptin levels of the whole series. (This figure appears in colour on the web.)

patients categorized according to the median value of plasma copeptin in the whole series. In univariate analysis, other variables associated with 3-month mortality were: previous ascites, previous HE, development of complications, serum bilirubin, INR, serum creatinine, serum sodium, leukocyte count, vasopressin levels, mean arterial pressure and Child-Pugh and MELD scores (Supplementary Table 3).

Finally, a logistic regression model was created to identify the independent predictive factors of 3-month mortality. In order to avoid collinearity with Child-Pugh and MELD scores, individual variables included in these scores were not included in the model. The model showed that plasma copeptin together with MELD score and leukocyte count was an independent predictive 


\section{Research Article}

factor of 3-month mortality (AUROC 0.860; 95\% CI [0.811-0.909]) (Table 4).

An internal validation of the prognostic model was performed with the use of the bootstrapping technique. Logistic regression model for 3-month mortality was internally validated by means of a bootstrap re-estimation of odds ratio and its confidence intervals. The model was fitted on 10,000 samples obtained with replacement from the study population. Estimations from the original model were considered to be validated if they were within the limits of these intervals. Bootstrapping for the prognostic model shows the following results (odds ratio and 95\% CI): copeptin 1.654 (1.165-2.457), MELD score 1.179 (1.123$1.265)$ and leukocyte count 2.035 (1.160-4.100).

Table 4. Independent predictive factors of 90-day mortality. Multivariate analysis.

Model 1 Study population

\begin{tabular}{llll}
\hline Variable & OR & $95 \% \mathrm{Cl}$ & $p$ value \\
\hline Copeptin & 1.664 & $1.140-2.429$ & 0.008 \\
MELD score & 1.191 & $1.123-1.264$ & $<0.0001$ \\
Leukocyte count & 1.951 & $1.095-3.477$ & 0.023 \\
\hline
\end{tabular}

AUROC $0.860 ; 95 \%$ Cl (0.811-0.909)

Model 2 Validation series

\begin{tabular}{llll}
\hline Variable & OR & $95 \% \mathrm{Cl}$ & $p$ value \\
\hline Copeptin & 2.03 & $1.140-3.60$ & 0.016 \\
MELD score & 1.15 & $1.070-1.230$ & $<0.001$ \\
\hline
\end{tabular}

AUROC $0.827 ; 95 \% \mathrm{Cl}(0.743-0.910)$

Table 5. Demographic and baseline liver and kidney function of the $\mathbf{1 2 0}$ patients included in the validation series.

\begin{tabular}{lll}
\hline Variable & & \\
\hline Age (years) & $57 \pm 11$ & $(24-84)$ \\
Gender, male & $72(60 \%)$ & \\
\hline Etiology of cirrhosis: & & \\
$\quad$ Alcohol & $57(51 \%)$ & \\
Hepatitis C & $21(19 \%)$ & \\
Other & $34(30 \%)$ & \\
\hline Previous ascites & $85(71 \%)$ & \\
Previous hepatic encephalopathy & $48(40 \%)$ & \\
Shock at admission & $2(2 \%)$ & \\
Serum bilirubin (mg/dl) & $7.0 \pm 9.5$ & $(0.4-55)$ \\
Serum albumin (g/L) & $29 \pm 6$ & $(14-44)$ \\
INR & $1.7 \pm 0.6$ & $(1.0-4.4)$ \\
Child-Pugh score & $10 \pm 2$ & $(5-15)$ \\
MELD score & $20 \pm 8$ & $(6-40)$ \\
Serum creatinine (mg/dl) & $1.4 \pm 1.1$ & $(0.5-6.8)$ \\
Serum sodium (mEq/L) & $135 \pm 6.2$ & $(115-149)$ \\
Leukocyte count (109 cells/L) & $7.2 \pm 5.5$ & $(1.3-41)$ \\
Mean arterial pressure (mmHg) & $82 \pm 14$ & $(47-131)$ \\
Copeptin (pmol/L) & 19 & $(10-44)$ \\
\hline
\end{tabular}

Data are expressed as mean \pm SD and ranges or number and percentages, except for copeptin which is expressed as median (interquartile range).

\section{External validation}

To validate the role of copeptin as an independent predictive factor of mortality, we analyzed an independent cohort of patients from different European centers. A subgroup of 120 patients was randomly selected from patients included in the CANONIC Study [28]. Eligibility criteria for patients from the validation series were the same as those for the study cohort. Baseline clinical and liver and kidney function tests of the validation series are shown in Table 5. Overall, median plasma copeptin levels were $19 \mathrm{pmol} / \mathrm{L}$ (IQR 10-44). A logistic regression model was performed to evaluate the best independent predictive factors of 3 -month mortality in this series. Results were comparable to those obtained in the study population, showing that plasma copeptin was an independent predictive factor of 3-month mortality (AUROC 0.827; 95\% CI [0.743-0.910]) (Table 4).

\section{Discussion}

The main finding of the current study is that plasma copeptin is an independent predictive factor of acute decompensation and mortality in patients with cirrhosis. The role of copeptin as prognostic biomarker has been investigated in several acute and chronic diseases such as heart failure, chronic respiratory diseases or sepsis [14-20]; however, information in cirrhosis is very limited. To our knowledge, there is only one study specifically assessing the role of copeptin in patients with cirrhosis [21]. This study showed that there was a significant correlation between plasma copeptin levels and the severity of liver disease, as defined by Child-Pugh and MELD score, and that copeptin was an independent predictive factor of 1-year mortality [21]. Nevertheless, the study was performed in a low number of patients and did not provide information on the relationship between copeptin and systemic hemodynamics and disease progression.

Copeptin is the C-terminal fragment of the vasopressin precursor and is secreted from the neurohypophysis together with AVP $[9,10]$. Patients with decompensated cirrhosis show high levels of AVP in response to hemodynamic stimulus due to the existence of effective arterial hypovolemia secondary to splanchnic arterial vasodilation and portal hypertension [2,3]. Although AVP levels could represent a biomarker of advanced cirrhosis, studies on AVP are very limited due to the instability of the molecule making its measurement difficult and unsuitable as biomarker. In this context, copeptin as a reliable surrogate marker of AVP has interest as potential biomarker of cirrhosis progression.

In keeping with the results of previous pathophysiological studies in healthy subjects or disorders of AVP secretion [11-13], our results indicate that there is a significant correlation between plasma AVP and copeptin levels in patients with cirrhosis, a condition characterized by extracellular fluid volume expansion and increased AVP secretion. Besides correlation with AVP, plasma copeptin levels showed a significant correlation with endogenous vasoactive systems, measured by PRA, aldosterone, and noradrenaline. Therefore, these results indicate that copeptin clearly reflects the progressive circulatory dysfunction that develops in patients with advanced cirrhosis as a good surrogate marker of AVP.

Besides its relationship with circulatory dysfunction, our results show that plasma copeptin levels progressively increase with the impairment of liver function as assessed by MELD score. Moreover, patients with previous history of decompensation of 
cirrhosis had significantly higher copeptin levels compared to patients with compensated cirrhosis.

Nevertheless, the most novel and main result of our study is that copeptin is not only related with the impairment of liver function but also with clinical outcomes. Patients developing complications of the disease and those who died during followup showed higher baseline plasma copeptin levels compared to those of patients who did not develop complications and remained compensated. Finally, and most interestingly, in the multivariate analysis, plasma copeptin was an independent predictive factor of the development of complications of cirrhosis and 3-months mortality. Moreover, the performance of the predictive model of mortality was validated in an independent cohort of patients from other centers.

Assessment of prognosis in patients with cirrhosis has been based on standard liver tests [29]. Several variables, such as MELD score and serum sodium, have been described as prognostic factors in cirrhosis in terms of mortality [30-32]. Other nonliver specific parameters such as inflammatory biomarkers (i.e., C-reactive protein) or vasoactive molecules (i.e., adrenomedullin, cortisol) have also been shown to be related with severity of cirrhosis and mortality in some studies [33-35]. However, to our knowledge there are no studies specifically assessing predictive factors of acute decompensation in patients with cirrhosis. Although MELD score is already known as a good and accurate variable to assess prognosis, results of the present study show that copeptin has some prognostic value independent of MELD score. In view of the results of our study, copeptin appears to be a reliable biomarker of disease progression and clinical decompensation in patients with cirrhosis. Considering these results we can hypothesize that its accuracy in predicting complications and prognosis may be related to the capacity of copeptin to reflect circulatory disturbances in patients with advanced cirrhosis, which are responsible, at least in part, for development of complications of the disease. In this context and considering that copeptin is a stable molecule and much easier to measure than AVP, it should be pointed out that copeptin is a better biomarker of disease progression and prognosis compared to vasopressin. Therefore, we believe that copeptin could be helpful in the assessment of prognosis of patients with cirrhosis. Increased plasma copeptin levels indicate that a patient is at increased risk of developing complications in the following months. This would be important information for clinicians in order to monitor these patients more strictly to prevent or early diagnose potential complications.

We also investigated the relationship between copeptin and hyponatremia and kidney function. It is well-known that nonosmotic secretion of AVP is the key mechanism of hyponatremia in patients with cirrhosis $[2,36]$. Therefore, considering the good correlation between copeptin, AVP, and circulatory function it would be expected that plasma copeptin levels could also play a role as biomarker of hyponatremia. Our results show an inverse and significant correlation between copeptin and serum sodium levels. Moreover, patients with hyponatremia showed higher plasma copeptin levels compared to those of patients with normal serum sodium. However, plasma copeptin levels were not able to identify those patients with increased risk of development of hyponatremia during follow-up. Although AVP seems to play a key role, the pathophysiology of hyponatremia in cirrhosis is complex and multifactorial. Other factors such as glomerular filtration rate and renal prostaglandin E2 production are also

\section{JOURNAL OF HEPATOLOGY}

involved [2]. Therefore, the effect of other factors different from AVP in the impairment of solute-free water retention in cirrhosis may be the reason to explain the lack of accuracy of copeptin in predicting development of hyponatremia. With respect to kidney function, our results show that there is a significant correlation between copeptin and kidney function, as estimated by serum creatinine levels. Moreover, patients who developed kidney failure during follow-up showed significantly higher baseline plasma copeptin levels compared to patients who did not develop kidney failure. To our knowledge, there is no previously reported information on the relationship between copeptin and kidney function. Copeptin is a small molecule with a molecular weight of 5 KDa [10]. Therefore, as it is filtered by the kidney its levels may be influenced by changes in GFR. In this context, it could be hypothesized that high plasma copeptin levels in patients with cirrhosis and kidney failure could be explained, at least in part, by the reduction in GFR.

Finally, it should be considered that this is a single center study and this could represent a limitation in order to extrapolate the results. However, the main results of the study have been successfully validated in an independent cohort of patients from other centers. There were few missing values in some of the variables of the study, which could represent a limitation for the analysis. However, it should be noted that missing values for the most important variables was very low so that this may not have had an impact on the results.

In conclusion, the results of the present study indicate that copeptin is a novel and reliable biomarker of disease progression, clinical decompensation, and prognosis in patients with cirrhosis. Results suggest that copeptin reflects the progressive impairment of circulatory function that occurs in patients with advanced cirrhosis, as a good surrogate marker of AVP.

\section{Financial support}

The work discussed in this paper has been funded by grants FIS PI12/00330 and FIS08/0126 integrated in the Plan Nacional I+D $+\mathrm{I}$ and co-funded by ISCIII-Subdirección General de Evaluación and European Regional Development Fund. CIBEREHD is funded by the Instituto de Salud Carlos III. Additional support was obtained from Thermofisher Scientific Inc (Hennigsdorf, Germany). Part of this work (validation series) has been supported by the EASL-CLIF Consortium and European Foundation for the Study of Chronic Liver Failure (EF-CLIF) which is supported by an unrestricted grant from Grifols. Isabel Graupera was supported by a grant from Instituto de Salud Carlos III (Rio Hortega fellowship). Pere Ginès is recipient of an Institució Catalana de Recerca i Estudis Avançats (ICREA) Academia Award.

\section{Conflict of interest}

Rajiv Jalan is consultant to Ocera Therapeutics and Conatus Pharmaceuticals and has received research grants from Grifols, Gambro, Sequana Medical and Ocera Therapeutics. François Durand is consultant for Novartis, Gilead, BMS, Astellas and has received research grants from Gilead and Astellas. Pere Ginès received financial support from Thermofisher Scientific Inc and research grants from Grifols and Sequana Medical. Other authors have nothing to disclose. 
Research Article

\section{Authors' contributions}

The authors listed above have all contributed to this manuscript and approve the version of this submission. ES and JK equally contributed to the conception and design of the study, acquisition of data, the analysis and interpretation of the data and drafting the manuscript; HV, RM, RC, EP and AA participated in acquisition of data and the analysis and interpretation of the data and statistical analysis as well as in the critical revision of the paper; PR, MM, EL, IG, CS, PH, XA, RJ, NF, DB, GP, FD, WJ, JR, JF and BH participated in the generation and collection of data, assembly of data, analyses of the results, interpretation of data, and/or critical revision of the manuscript for important intellectual content. MC and PG participated in the study concept, interpretation of the data, drafting the manuscript, critical revision of the manuscript for important intellectual content, obtained funding and study supervision.

\section{Acknowledgements}

We are indebted to the IDIBAPS Biobank, Barcelona, Spain, for sample procurement and to Nicki van Berckel for her administrative support.

\section{Supplementary data}

Supplementary data associated with this article can be found, in the online version, at http://dx.doi.org/10.1016/j.jhep.2016.07. 003.

\section{References}

[1] Ginès P, Quintero E, Arroyo V, et al. Compensated cirrhosis: natural history and prognostic factors. Hepatology 1987;7:122-128.

[2] Ginès P, Cárdenas A, Solà E, Schrier RW. Liver Disease and the Kidney. In: Schrier RW, editor. Schrieŕs Diseases of the kidney. Coffman TM, Falk RJ, Molitoris BA, Neilson EG, Schrier RW, Eds. 9th ed. Lippincott Williams \& Wilkins; 2012.

[3] Schrier RW, Arroyo V, Bernardi M, Epstein M, Henriksen JH, Rodés J. Peripheral arterial vasodilatation hypothesis: a proposal for the initiation of renal sodium and water retention in cirrhosis. Hepatology 1988;8: $1151-1157$.

[4] Kluge M et al. Improved extraction procedure and RIA for determination of arginine-vasopressin in plasma: role of premeasurement sample treatment and reference values in children. Clin Chem 1999;45:98-103.

[5] Baumann G, Dingman JF. Distribution, blood transport, and degradation of antidiuretic hormone in man. J Clin Invest 1976;57:1109-1116.

[6] Robertson GL et al. Development and clinical application of a new method for the radioimmunoassay of arginine vasopressin in human plasma. J Clin Invest 1973;52:2340-2352.

[7] Holwerda DA. A glycopeptide from the posterior lobe of pig pituitaries. I. Isolation and characterization. Eur J Biochem 1972;28:334-339.

[8] Land $\mathrm{H}$ et al. Nucleotide sequence of cloned cDNA encoding bovine arginine vasopressin-neurophysin II precursor. Nature 1982;295:299-303.

[9] Morgenthaler LG, Struck J, Jochberger S, Dünser MV. Copeptin: clinical use of a new biomarker. Trends Endocrinol Metab 2008;19:43-49.

[10] Morgenthaler LG, Struck J, Alonso C, Bergamann A. Assay for the measurement of copeptin, a stable peptide derived from the precursor of vasopressin. Clin Chem 2006;52:112-119.

[11] Bhandari SS, Loke I, Davies JE, et al. Gender and renal function influences plasma levels of copeptin in healthy individuals. Clin Sci 2009;116:257-263.

[12] Szinnai G, Morgenthaler NG, Berneis K, et al. Changes in plasma copeptin, the C-terminal portion of arginine-vasopressin during water deprivation and excess in healthy subjects. J Clin Endocrinol Metab 2007;92: 3973-3978.

[13] Balanescu S, Kopp P, Gaskill MB, et al. Correlation of plasma copeptin and vasopressin concentrations in hypo-, iso-, and hyperosmolar states. J Clin Endocrinol Metab 2011;96:1046-1052.

[14] Miller WL, Hartamn KA, Grill DE, et al. Serial measurements of midregion proANP and copeptin in ambulatory patients with heart failure: incremental prognostic value of novel biomarkers in heart failure. Heart 2012;98:389-394.

[15] Maisel A, Xue Y, Shah K, et al. Increased 90-day mortality in patients with acute heart failure with elevated copeptin secondary results from the Biomarkers in Acute Heart Failure (BACH) study. Circ Heart Fail 2011;4:613-620.

[16] Khan SQ, Dhillon OS, Quinn PA, et al. C-terminal provasopressin (copeptin) as a novel and prognostic marker in acute myocardial infarction: Leicester Acute Myocardial Infarction Peptide (LAMP) study. Circulation 2007; 115:2103-2110.

[17] Morgenthaler NG, Müller B, Struck J, et al. Copeptin, a stable peptide of the arginine vasopressin precursor, is elevated in hemorrhagic and septic shock. Shock 2007:28:219-226.

[18] Jochberger S, Morgenthaler NG, Mayr VD. Copeptin and arginine vasopressin concentrations in critically ill patients. J Clin Endocrinol Metab 2006;91:4381-4386.

[19] Lesur O, Roussy JF, Chagnon F, et al. Proven infection-related sepsis induces a differential stress response early after ICU admission. Crit Care 2010;14: R131.

[20] Stolz D, Christ-Crain M, Morgenthaler NG, et al. Copeptin, C-reactive protein, and procalcitonin as prognostic biomarkers in acute exacerbation of COPD. Chest 2007:131:1058-1067.

[21] Moreno JP, Grandclement E, Monnet E, et al. Plasma copeptin, a possible prognostic marker in cirrhosis. Liver Int 2013;33:843-851.

[22] Pereira GH, Guevara M, Fagundes C, et al. Renal failure and hyponatremia in patients with cirrhosis and skin and soft tissue infection. A retrospective study. J Hepatol 2012;56:1040-1046.

[23] Follo A, Lovet JM, Navasa M, et al. Renal impairment after spontaneous bacterial peritonitis in cirrhosis: incidence, clinical course, predictive factors and prognosis. Hepatology 1994;20:4095-4501.

[24] Barreto R, Elia C, Solà E, et al. Urinay neutrophil-gelatinase associated lipocalin predicts kidney outcome and death in patients with cirrhosis and bacterial infections. J Hepatol 2014:61:35-42.

[25] Uriz J, Ginès P, Cárdenas A. Terlipressin plus albumin infusion: an effective and safe therapy of hepatorenal syndrome. J Hepatol 2000;33:43-48.

[26] Guevara M, Ginès P, Bandi JC, et al. Transjugular intrahepatic portosystemic shunt in hepatorenal syndrome: effects on renal function and vasoactive systems. Hepatology 1998;28:416-422.

[27] Moons K, Altman D, Reitsma J, et al. Transparent reporting of a multivariable prediction model for individual prognosis or diagnosis (TRIPOD): explanation and elaboration. Ann Intern Med 2015;162:W1-W73.

[28] Moreau R, Jalan R, Ginès P, et alCANONIC Study Investigators of the EASLCLIF Consortium. Acute-on-chronic liver failure is a distinct syndrome that develops in patients with acute decompensation of cirrhosis. Gastroenterology 2013;144:1426-1437.

[29] Cárdenas A, Solà E, Graupera I, Ginès P. Cirrhosis and its complications. In: ACP Medicine. American College of Physicians, 2013.

[30] Kamath PS, Kim R. Advanced Liver Disease Study Group. The model for endstage liver disease (MELD). Hepatology 2007;45:797-805.

[31] Angeli P, Wong F, Watson H, et al. Hyponatremia in cirrhosis: results of a patient population survey. Hepatology 2006;44:1535-1542.

[32] Llach J, Ginès P, Arroyo V, et al. Prognostic value of arterial pressure, endogenous vasoactive systems, and renal function in cirrhotic patients admitted to the hospital for the treatment of ascites. Gastroenterology 1988;94:482-487.

[33] Cervoni JP, Theventot T, Weil D, et al. C-reactive protein predicts short-term mortality in patients with cirrhosis. J Hepatol 2012;56:1299-1304.

[34] Theventot T, Dorin R, Monnet E, et al. High serum levels of free cortisol indicate severity of cirrhosis in haemodynamically stable patients. J Gastroenterol Hepatol 2012;27:1597-1601.

[35] Guevara M, Ginès P, Jimenez W, et al. Increased adrenomedullin levels in cirrhosis: relationship with haemodynamic abnormalities and vasoconstrictor systems. Gastroenterology 1998;114:336-343.

[36] Ginès P, Guevara M. Hyponatremia in cirrhosis: pathogenesis, clinical significance and management. Hepatology 2008;48:1002-1010. 